\title{
Clinical and angiographic correlation of high-sensitivity C-reactive protein with acute ST elevation myocardial infarction
}

\author{
SYED TANVEER $^{1}$, SHAHEENA BANU ${ }^{2}$, NASIMUDEEN REHUMATHBEEVI JABIR ${ }^{3}$, MOHD SHAHNAWAZ KHAN ${ }^{4}$, \\ GHULAM MD ASHRAF ${ }^{3}$, NANJAPPA CHOLENAHALLY MANJUNATH ${ }^{1}$ and SHAMS TABREZ $^{3}$

\begin{abstract}
Departments of ${ }^{1}$ Cardiology and ${ }^{2}$ Biochemistry, Sri Jayadeva Institute of Cardiovascular Science and Research,
${ }^{4}$ Department of Biochemistry, College of Science, King Saud University, Riyadh 11451, Kingdom of Saudi Arabia
\end{abstract} \\ Bangalore, Karnataka 560069, India; ${ }^{3}$ King Fahd Medical Research Center, King Abdulaziz University, Jeddah 21589;
}

Received July 28, 2015; Accepted September 15, 2016

DOI: $10.3892 / \mathrm{etm} .2016 .3882$

\begin{abstract}
Vascular inflammation and associated ongoing inflammatory responses are considered as the critical culprits in the pathogenesis of acute atherothrombotic events such as acute coronary syndrome (ACS) and myocardial infarction (MI). ST segment elevation myocardial infarction (STEMI) is considered as one of the prominent clinical forms of ACS. Moreover, C-reactive protein (CRP) is an important acute phase prsotein, which may be estimated using high-sensitivity methods (hs-CRP), and its elevated level in body fluids reflects chronic inflammatory status. The circulating hs-CRP level has been proposed as a promising inflammatory marker of coronary artery disease (CAD). The present study investigated the correlation of hs-CRP level with clinical and angiographic features of STEMI, various other traditional risk factors, complications of myocardial infarction and angiographically significant CAD. Out of 190 patients with STEMI that were analyzed, the interval between symptom onset and reperfusion therapy (window period) varied from 0.5 to $24 \mathrm{~h}$. The hs-CRP value was found to be higher in non-diabetic patients $(0.61 \mathrm{mg} / \mathrm{dl})$ compared with diabetic patients $(0.87 \mathrm{mg} / \mathrm{dl})$. Moreover, a significant correlation between hs-CRP and hs-troponin $\mathrm{T}$ was also recorded $(\mathrm{P}<0.001)$. However, there was no significant difference in the mean hs-CRP values in patients with or without mortality. It is considered that the present study will increase the understanding of atherosclerosis in general and may also have clinical applications in the targeting of therapy for this harmful disease.
\end{abstract}

Correspondence to: Dr Shams Tabrez, King Fahd Medical Research Center, King Abdulaziz University, PO Box 80216, Jeddah 21589, Kingdom of Saudi Arabia

E-mail: shamstabrez1@gmail.com

Key words: high-sensitivity C-reactive protein, ST elevation, Syntax score, myocardial infarction, coronary artery disease, diabetes

\section{Introduction}

Over the past several years, it has become clear that inflammatory processes regulate atherosclerotic disease at a fundamental level (1). Fewer than 50\% of cases of coronary artery disease (CAD) can be ascribed to traditional risk factors and the remaining cases are unexplained (2).

C-reactive protein (CRP) has emerged as the leading candidate marker for systemic inflammation, because of its predictive ability, wide availability, low cost and ease of use (3). It is a calcium-binding pentameric protein consisting of five identical, non-covalently linked, 23-kD subunits produced in the liver in response to interleukin (IL)-6, IL-1 and tumor necrosis factor. CRP levels begin to rise within 4-6 $\mathrm{h}$ after tissue injury and can continue to rise exponentially to increase several hundred fold within 24-48 h. Moreover, the half-life of CRP is $<24 \mathrm{~h}(1,3)$. The predictive power of CRP for vascular risk detection is in the range of 0.1 to $0.5 \mathrm{mg} / \mathrm{dl}$, a level found in many healthy individuals without inflammation (4). Hence, CRP assays are required to be highly sensitive High-sensitivity CRP (hs-CRP) has a limit of detection as low as $0.02 \mathrm{mg} / \mathrm{dl}$ and is a well standardized assay $(5,6)$. Various trials have shown that the predictive value of hs-CRP is significantly higher compared with those of other traditional cardiovascular risk markers $(7,8)$.

The purpose of the present study was to investigate the association of hs-CRP with acute ST elevation myocardial infarction (STEMI), and its correlation with various other traditional risk factors, complications of myocardial infarction (MI) and angiographically significant CAD.

\section{Materials and methods}

Patients. The study included 190 consecutive patients with a diagnosis of acute STEMI admitted to the Coronary Care Unit (CCU) at Sri Jayadeva Institute of Cardiovascular Sciences and Research (Bangalore, India). The cross-sectional study was carried out between December 2012 and April 2013. The study was conducted in accordance with the Declaration of Helsinki and with approval from the ethics committee of Sri Jayadeva Institute of Cardiovascular Sciences and Research. Informed consent was obtained from all individual participants. 
The diagnosis of acute MI was made on the basis of the Joint ESC/ACCF/AHA/WHF Task Force's Third Universal Definition (9). Patients with diabetes mellitus had a fasting plasma glucose concentration $>126 \mathrm{mg} / \mathrm{dl}$, glycated hemoglobin level $>6.5 \%$, or were receiving treatment with insulin or hypoglycemic agents. Patients who had a systolic/diastolic blood pressure $>140 / 90 \mathrm{mmHg}$ or were currently using any antihypertensive medication were defined as hypertensive. Cigarette smoking was defined as active smoking in the last 6 months. Patients diagnosed with unstable angina and NSTEMI, were excluded from the study. According to a previously designed questionnaire, data included gender, age, body mass index (BMI), blood pressure and assessment of risk factors, including a history of MI. During baseline evaluation, symptoms were assessed, Killip class was assessed, and a thrombosis in MI (TIMI) score was calculated for all patients (10). Blood samples were drawn from patients at the time of admission to the CCU for the analysis of random blood glucose (RBS), renal function, serum electrolytes, lipid parameters, complete blood count, high-sensitivity (hs)-troponin T and hs-CRP. An echocardiographic evaluation of the left ventricular ejection fraction (LVEF) was performed in all participants within $24 \mathrm{~h}$ of hospital admission. Blood samples were processed within $24 \mathrm{~h}$ using automated micro-particle immunoassays (ELISA Immuno Explorer Kit; Bio-Rad Laboratories, Inc., Hercules, CA, USA). The hs-CRP detection range was $0.1-12.0 \mathrm{mg} / \mathrm{dl}$, with an inter-assay variation coefficient of $<5 \%$.

Angiographic assessment of coronary atherosclerosis. Two experienced cardiologists with no prior knowledge of the patients' biochemical results reviewed all angiographic images to assess the extent of CAD and morphology of all coronary artery stenosis. A significant CAD was defined based on the presence of $>50 \%$ coronary artery stenosis in any coronary artery or left main artery. A Syntax score was calculated for all coronary angiograms (CAGs) using on an online Syntax score evaluating algorithm version 2.1 (www.syntaxscore. com). Patients were divided into three groups on the basis of Syntax score: Low Syntax score (0-22 points), intermediate Syntax score (23-31 points) and high Syntax score ( $\geq 33$ points), and mean hs-CRP values were compared among these three groups (11).

Statistical analysis. All statistical analyses were performed using SPSS version 16.0 software (SPSS Inc., Chicago, IL, USA). Baseline characteristics were assessed with the Student's t-test (parametric) and Mann-Whitney U test (non-parametric) for continuous variables, and the $\chi^{2}$ test for categorical variables, with two-tailed P-values. $\mathrm{P}<0.05$ was considered to indicate a statistically significant difference.

\section{Results}

Baseline characteristics. Baseline characteristics of the study population comprising 190 patients with STEMI were analyzed. The patient age ranged from 20 to 90 years (mean, 54.37 years), as shown in Table I. Among those 190 patients, $150(79 \%)$ were males and $40(21 \%)$ were females. Anterior wall myocardial infarction (AWMI) was found to be the most common type of MI, present in 94 patients (49.5\%). Lateral wall
MI (LWMI) was found to be the least common type, present in only $0.5 \%$ of patients (Table II). The interval between symptom onset and reperfusion therapy (window period) varied from 0.5 to $24 \mathrm{~h}$, with a mean value of $5.59 \mathrm{~h}$ (Table I). Coronary risk factor frequencies such as hypertension were present in $37 \%$, type 2 diabetes mellitus in $38 \%$ and dyslipidemia in $83 \%$ of patients (Table II). Patients with hs-CRP $\geq 0.5 \mathrm{mg} / \mathrm{dl}$ had a higher mean age $(\mathrm{P}=0.029)$.

hs-CRP and various risk factors. There was no correlation between hs-CRP and various lipid parameters, serum creatinine, RBS, white blood cell count and platelet count (Table III). However, the mean values of hs-CRP were found to be higher in patients with hypertension, smoking and high total cholesterol (Table II). A significant difference in hs-CRP values was recorded between patients with diabetes and those without diabetes $(\mathrm{P}=0.029)$. $\mathrm{RBS}$ also had a positive correlation with hs-CRP values $(\mathrm{r}=0.165, \mathrm{P}=0.023$; Table III); however, no statistically significant difference in the mean value of hs-CRP was recorded between patients with normal and abnormal RBS. With regards to total cholesterol, mean hs-CRP values were found to be higher in patients with total cholesterol levels $>200 \mathrm{mg} / \mathrm{dl}$ (mean hs-CRP values 0.66 vs. $0.76 \mathrm{mg} / \mathrm{dl}$; $\mathrm{P}=0.068$; Table II). A significant correlation between hs-CRP and hs-troponin $\mathrm{T}(\mathrm{r}=0.306, \mathrm{P}<0.001$; Table III) was also recorded.

hs-CRP and clinical factors. A significant difference was observed in the mean hs-CRP values with regard to duration of the window period (Table I). Out of 190 patients, 141 patients were found to have a window period between 0 and $6 \mathrm{~h}$; among them 77 patients had hs-CRP levels $<0.5 \mathrm{mg} / \mathrm{dl}$ and 64 patients had hs-CRP levels $\geq 0.5 \mathrm{mg} / \mathrm{dl}$. Moreover, 49 patients presented with a window period of $>6 \mathrm{~h}$, with 18 patients having hs-CRP values $<0.5 \mathrm{mg} / \mathrm{dl}$, and 31 patients having hs-CRP values $\geq 0.5 \mathrm{mg} / \mathrm{dl}(\mathrm{P}=0.05)$.

Table II shows that among the different types of MI, there was no statistically significant difference in the mean hs-CRP values. However, patients presenting with combined AWMI and IWMI had the highest hs-CRP values, whereas patients with IWMI had lowest hs-CRP values (1.29 vs. $0.54 \mathrm{mg} / \mathrm{dl})$. Over all, $\sim 50 \%$ of STEMI patients had hs-CRP elevation $(>0.5 \mathrm{mg} / \mathrm{dl})$ and the remaining $50 \%$ of patients had hs-CRP levels within normal limits $(<0.5 \mathrm{mg} / \mathrm{dl})$. With regard to left ventricular ejection fraction (LVEF), no statistically significant difference in hs-CRP values was recorded among patients with different LVEF percentages; however patients with an LVEF $<30 \%$ had a higher mean hs-CRP value $(0.76 \mathrm{mg} / \mathrm{dl})$ compared with patients with an LVEF $>50 \%(0.48 \mathrm{mg} / \mathrm{dl})$. There was no significant correlation between hs-CRP and TIMI score (Table III), although higher TIMI scores were associated with higher hs-CRP values. There was a significant difference in hs-CRP values among patients with different Killip classes $(\mathrm{P}<0.029)$, with higher mean hs-CRP values observed in patients with higher Killip classes (Table II).

hs-CRP and coronary angiography. Following coronary angiography, single, double and triple vessel CAD was identified in 43, 25 and 7 patients, respectively (Table IV). Tables IV-VI show the associations between hs-CRP and CAG findings. 
Table I. Patient data and window period.

\begin{tabular}{lrrrrr}
\hline Parameter & Mean & SD & SE of mean & Median & Min \\
\hline Age (years) & 54.37 & 11.73 & 0.85 & 55 & 20 \\
Weight $(\mathrm{kg})$ & 59.66 & 8.55 & 0.62 & 58 & 90 \\
Height $(\mathrm{cm})$ & 155.25 & 4.35 & 0.32 & 155 & 45 \\
BMI $\left(\mathrm{kg} / \mathrm{m}^{2}\right)$ & 24.67 & 2.69 & 0.20 & 24.44 & 148 \\
Window period (h) & 5.59 & 3.70 & 0.27 & 5 & 19.98 \\
\end{tabular}

BMI, body mass index; SD, standard deviation; SE, standard error.

Table II. Patient clinical parameters.

\begin{tabular}{|c|c|c|c|c|c|c|c|c|c|}
\hline \multirow[b]{2}{*}{ Parameter } & \multirow[b]{2}{*}{$\mathrm{N}$} & \multirow[b]{2}{*}{$\begin{array}{l}\text { Mean hs-CRP } \\
(\mathrm{mg} / \mathrm{dl})\end{array}$} & \multirow[b]{2}{*}{$\mathrm{SD}$} & \multirow[b]{2}{*}{$\begin{array}{l}\text { SE of } \\
\text { mean }\end{array}$} & \multicolumn{2}{|c|}{$95 \% \mathrm{CI}$ for mean } & \multirow[b]{2}{*}{ Min } & \multirow[b]{2}{*}{$\operatorname{Max}$} & \multirow[b]{2}{*}{ P-value } \\
\hline & & & & & $\begin{array}{l}\text { Lower } \\
\text { bound }\end{array}$ & $\begin{array}{l}\text { Upper } \\
\text { bound }\end{array}$ & & & \\
\hline MI type & & & & & & & & & 0.279 \\
\hline AWMI & 94 & 0.67 & 0.82 & 0.08 & 0.51 & 0.84 & 0.02 & 4.67 & \\
\hline AWMI + LWMI & 4 & 0.98 & 1.22 & 0.61 & -0.97 & 2.92 & 0.06 & 2.77 & \\
\hline IWMI & 26 & 0.54 & 0.48 & 0.09 & 0.35 & 0.74 & 0.01 & 1.50 & \\
\hline IWMI + RVMI + PWMI & 13 & 0.65 & 0.53 & 0.15 & 0.33 & 0.97 & 0.05 & 1.60 & \\
\hline IWMI + PWMI & 26 & 0.92 & 0.94 & 0.18 & 0.54 & 1.30 & 0.06 & 4.98 & \\
\hline IWMI + RWMI & 22 & 0.73 & 0.79 & 0.17 & 0.38 & 1.08 & 0.02 & 3.34 & \\
\hline AWMI + IWMI & 3 & 1.29 & 1.14 & 0.66 & -1.54 & 4.11 & 0.30 & 2.53 & \\
\hline LWMI & 1 & 0.85 & - & - & - & - & 0.85 & 0.85 & \\
\hline Hypertension & & & & & & & & & 0.181 \\
\hline Absent & 120 & 0.69 & 0.87 & 0.08 & 0.54 & 0.85 & 0.01 & 4.98 & \\
\hline Present & 70 & 0.73 & 0.64 & 0.08 & 0.58 & 0.88 & 0.02 & 3.34 & \\
\hline Diabetes mellitus & & & & & & & & & 0.029 \\
\hline Absent & 118 & 0.61 & 0.71 & 0.07 & 0.48 & 0.74 & 0.01 & 4.98 & \\
\hline Present & 72 & 0.87 & 0.88 & 0.10 & 0.66 & 1.07 & 0.02 & 4.51 & \\
\hline Smoking & & & & & & & & & 0.818 \\
\hline Absent & 87 & 0.69 & 0.77 & 0.08 & 0.53 & 0.86 & 0.02 & 4.51 & \\
\hline Present & 103 & 0.72 & 0.81 & 0.08 & 0.56 & 0.88 & 0.01 & 4.98 & \\
\hline Dyslipidemia & & & & & & & & & 0.419 \\
\hline Absent & 32 & 0.87 & 0.90 & 0.16 & 0.54 & 1.19 & 0.02 & 3.34 & \\
\hline Present & 158 & 0.67 & 0.76 & 0.06 & 0.55 & 0.80 & 0.01 & 4.98 & \\
\hline LVEF (\%) & & & & & & & & & 0.453 \\
\hline$<30$ & 2 & 0.76 & 0.37 & 0.26 & -2.54 & 4.06 & 0.50 & 1.02 & \\
\hline $30-40$ & 51 & 0.81 & 0.90 & 0.13 & 0.55 & 1.06 & 0.01 & 4.51 & \\
\hline $41-50$ & 112 & 0.71 & 0.80 & 0.08 & 0.56 & 0.86 & 0.02 & 4.98 & \\
\hline$>50$ & 25 & 0.48 & 0.44 & 0.09 & 0.30 & 0.66 & 0.02 & 1.50 & \\
\hline Killip class & & & & & & & & & 0.029 \\
\hline Class I & 141 & 0.62 & 0.71 & 0.06 & 0.50 & 0.74 & 0.01 & 4.98 & \\
\hline Class II & 33 & 0.95 & 1.02 & 0.18 & 0.58 & 1.31 & 0.05 & 4.51 & \\
\hline Class III & 4 & 1.08 & 1.22 & 0.61 & -0.86 & 3.01 & 0.31 & 2.89 & \\
\hline Class IV & 12 & 0.98 & 0.63 & 0.18 & 0.59 & 1.38 & 0.22 & 2.26 & \\
\hline Total cholesterol & & & & & & & & & 0.068 \\
\hline Normal & 103 & 0.66 & 0.79 & 0.08 & 0.51 & 0.81 & 0.01 & 4.67 & \\
\hline Increased/risk & 87 & 0.76 & 0.79 & 0.09 & 0.59 & 0.93 & 0.02 & 4.98 & \\
\hline
\end{tabular}


Table II. Continued.

\begin{tabular}{|c|c|c|c|c|c|c|c|c|c|}
\hline \multirow[b]{2}{*}{ Parameter } & \multirow[b]{2}{*}{$\mathrm{N}$} & \multirow[b]{2}{*}{$\begin{array}{l}\text { Mean hs-CRP } \\
(\mathrm{mg} / \mathrm{dl})\end{array}$} & \multirow[b]{2}{*}{ SD } & \multirow[b]{2}{*}{$\begin{array}{l}\text { SE of } \\
\text { mean }\end{array}$} & \multicolumn{2}{|c|}{$95 \% \mathrm{CI}$ for mean } & \multirow[b]{2}{*}{ Min } & \multirow[b]{2}{*}{$\operatorname{Max}$} & \multirow[b]{2}{*}{ P-value } \\
\hline & & & & & $\begin{array}{l}\text { Lower } \\
\text { bound }\end{array}$ & $\begin{array}{l}\text { Upper } \\
\text { bound }\end{array}$ & & & \\
\hline HDL & & & & & & & & & 0.534 \\
\hline Risk & 118 & 0.68 & 0.82 & 0.08 & 0.53 & 0.83 & 0.01 & 4.98 & \\
\hline Normal & 72 & 0.75 & 0.74 & 0.09 & 0.58 & 0.92 & 0.02 & 3.34 & \\
\hline Non-HDL & & & & & & & & & 0.927 \\
\hline Normal & 59 & 0.68 & 0.77 & 0.10 & 0.48 & 0.88 & 0.01 & 4.67 & \\
\hline Risk & 131 & 0.72 & 0.80 & 0.07 & 0.58 & 0.86 & 0.02 & 4.98 & \\
\hline LDL & & & & & & & & & 0.202 \\
\hline Normal & 60 & 0.57 & 0.56 & 0.07 & 0.42 & 0.71 & 0.01 & 2.89 & \\
\hline Risk & 130 & 0.77 & 0.87 & 0.08 & 0.62 & 0.92 & 0.02 & 4.98 & \\
\hline TGL & & & & & & & & & 0.022 \\
\hline Normal & 83 & 0.88 & 0.95 & 0.10 & 0.67 & 1.09 & 0.02 & 4.98 & \\
\hline Risk & 107 & 0.57 & 0.61 & 0.06 & 0.46 & 0.69 & 0.01 & 4.51 & \\
\hline Hemoglobin & & & & & & & & & 0.617 \\
\hline Anemic & 47 & 0.72 & 0.73 & 0.11 & 0.51 & 0.94 & 0.02 & 3.34 & \\
\hline Normal & 143 & 0.70 & 0.81 & 0.07 & 0.57 & 0.84 & 0.01 & 4.98 & \\
\hline Total WBC count & & & & & & & & & 0.386 \\
\hline Normal & 111 & 0.70 & 0.83 & 0.08 & 0.54 & 0.85 & 0.01 & 4.98 & \\
\hline Abnormal & 79 & 0.72 & 0.73 & 0.08 & 0.56 & 0.89 & 0.02 & 4.51 & \\
\hline Platelet count & & & & & & & & & 0.649 \\
\hline Normal & 186 & 0.71 & 0.80 & 0.06 & 0.60 & 0.83 & 0.01 & 4.98 & \\
\hline Abnormal & 4 & 0.49 & 0.54 & 0.27 & -0.37 & 1.36 & 0.14 & 1.30 & \\
\hline RBS & & & & & & & & & 0.159 \\
\hline Normal & 80 & 0.66 & 0.83 & 0.09 & 0.47 & 0.84 & 0.01 & 4.98 & \\
\hline Abnormal & 110 & 0.74 & 0.76 & 0.07 & 0.60 & 0.89 & 0.02 & 4.51 & \\
\hline \multicolumn{10}{|l|}{ Urea } \\
\hline Normal & 177 & 0.69 & 0.77 & 0.06 & 0.57 & 0.80 & 0.01 & 4.98 & 0.625 \\
\hline Abnormal & 13 & 0.99 & 1.08 & 0.30 & 0.33 & 1.64 & 0.05 & 3.34 & \\
\hline \multicolumn{10}{|l|}{ Creatinine } \\
\hline Normal & 159 & 0.67 & 0.73 & 0.06 & 0.55 & 0.78 & 0.01 & 4.98 & 0.340 \\
\hline Abnormal & 31 & 0.91 & 1.05 & 0.19 & 0.52 & 1.29 & 0.05 & 4.67 & \\
\hline
\end{tabular}

hs-CRP, high-sensitivity C-reactive protein; SD, standard deviation; SE, standard error; CI, confidence interval; MI, myocardial infarction; AWMI, anterior wall MI; LWMI, lateral wall MI; IWMI, inferior wall MI; RVMI, right ventricular MI; PWMI, posterior wall MI; LVEF, left ventricular ejection fraction; HDL, high-density lipoprotein; LDL, low-density lipoprotein; TGL, triglycerides; WBC, white blood cell; RBS, random blood glucose.

Although there was no statistically significant difference in mean hs-CRP values among the single vessel disease (SVD), double vessel disease (DVD) and triple vessel disease (TVD) groups, hs-CRP values were higher in the DVD and TVD groups than in the SVD group (SVD, $0.56 \mathrm{mg} / \mathrm{ml}$ vs. DVD, $0.72 \mathrm{mg} / \mathrm{ml}$ and TVD, $0.97 \mathrm{mg} / \mathrm{ml}$ ). Similarly there was no significant difference in mean hs-CRP values with respect to the number of vessels involved individually, but mean hs-CRP values were found to be higher in patients with a higher number of coronary arteries involved ( 1 vessel, $0.54 \mathrm{mg} / \mathrm{ml} ; 2$ vessels, $0.68 \mathrm{mg} / \mathrm{ml}$; 3 vessels, $0.85 \mathrm{mg} / \mathrm{ml})$. Moreover, there was no significant difference in the mean hs-CRP values according to Syntax scores when analyzed individually, but mean hs-CRP values correlated positively with hs-CRP value; higher hs-CRP values were observed in patients with elevated Syntax scores [Syntax score $\leq 22$ (mild CAD), $0.59 \mathrm{mg} / \mathrm{ml}$ vs. Syntax scores $>22-32$ (moderate CAD), $0.89 \mathrm{mg} / \mathrm{ml}$ and $>32$ (severe CAD), $2.11 \mathrm{mg} / \mathrm{dl} ; \mathrm{r}=0.285, \mathrm{P}=0.007$; Tables III and IV]. Table VI shows the analysis of the combined study group according to hs-CRP value, where a high mean hs-CRP ( $\geq 5 \mathrm{mg} / \mathrm{dl}$ ) was found to be associated with a higher number of involved coronary arteries and higher Syntax score. There was a statistically 
Table III. Correlation analysis of hs-CRP and hs-TROP T.

\begin{tabular}{|c|c|c|c|c|}
\hline \multirow[b]{2}{*}{ Parameter } & \multicolumn{2}{|c|}{ hs-CRP (mg/dl) } & \multicolumn{2}{|c|}{ hs-Trop T (ng/ml) } \\
\hline & $\mathrm{r}$ & $\mathrm{P}$-value & $\mathrm{r}$ & P-value \\
\hline Age & 0.051 & 0.484 & 0.106 & 0.146 \\
\hline Weight & -0.030 & 0.681 & -0.020 & 0.786 \\
\hline Height & 0.018 & 0.805 & -0.014 & 0.844 \\
\hline BMI & -0.049 & 0.499 & -0.025 & 0.737 \\
\hline Window period & 0.061 & 0.404 & 0.249 & 0.001 \\
\hline Heart rate & 0.088 & 0.229 & 0.006 & 0.936 \\
\hline SBP & -0.061 & 0.406 & -0.070 & 0.339 \\
\hline DBP & -0.073 & 0.314 & -0.048 & 0.515 \\
\hline TIMI score & 0.131 & 0.073 & 0.192 & 0.008 \\
\hline LVEF & -0.129 & 0.076 & -0.170 & 0.019 \\
\hline Total cholesterol & 0.035 & 0.632 & 0.060 & 0.409 \\
\hline HDL & 0.065 & 0.373 & 0.199 & 0.006 \\
\hline Non-HDL & 0.023 & 0.757 & 0.020 & 0.789 \\
\hline LDL & 0.101 & 0.165 & 0.141 & 0.053 \\
\hline TGL & -0.138 & 0.057 & -0.170 & 0.019 \\
\hline Hemoglobin & 0.003 & 0.969 & -0.003 & 0.969 \\
\hline Total WBC count & -0.013 & 0.858 & 0.069 & 0.341 \\
\hline Platelet count & -0.049 & 0.498 & -0.136 & 0.061 \\
\hline RBS & 0.165 & 0.023 & -0.028 & 0.701 \\
\hline Urea & 0.153 & 0.035 & 0.246 & 0.001 \\
\hline Creatinine & 0.070 & 0.338 & 0.002 & 0.979 \\
\hline Vessel count & 0.161 & 0.136 & 0.217 & 0.043 \\
\hline Syntax score & 0.285 & 0.007 & 0.186 & 0.084 \\
\hline hs-CRP (mg/dl) & - & - & 0.306 & $<0.001$ \\
\hline hs-Trop T (ng/ml) & 0.306 & $<0.001$ & - & - \\
\hline
\end{tabular}

hs-CRP, high-sensitivity C-reactive protein; hs-TROP T, high-sensitivity troponin T; BMI, body mass index; SBP, systolic blood pressure; DBP, diastolic blood pressure; TIMI, thrombosis in myocardial infarction; LVEF, left ventricular ejection fraction; HDL, high-density lipoprotein; LDL, low-density lipoprotein; TGL, triglycerides; WBC, white blood cell; RBS, random blood glucose.

significant difference in involved vessel number and Syntax score between the groups with low and high hs-CRP values $(\mathrm{P}=0.009$ and $\mathrm{P}=0.019$, respectively).

hs-CRP and complications. Table VII depicts the hs-CRP levels in patients with and without various in-hospital complications. No significant difference was identified in mean hs-CRP values among groups with and without various complications, with the exception of MI patients who had developed mitral regurgitation. However, the mean value of hs-CRP was found to be higher in the patients with LV dysfunction, heart failure, shock, bradyarrhythmias, tachyarrythmias and recurrent angina than in the patients without these complications.

$h s$-CRP and mortality. Tables IV and VIII show that 16 patients out of 190 patients succumbed during their in-hospital stay and 1 patient succumbed at home, 20 days post discharge. There was no significant difference in mean hs-CRP values in patients with or without mortality.

\section{Discussion}

Elevated CRP has been suggested as an effective parameter for identifying the risk of future ischemic events in patients with acute coronary syndrome (ACS). Biasucci et al (12) reported that patients with a CRP level $>0.3 \mathrm{mg} / \mathrm{dl}$ had a $>8$-fold higher risk of recurrent ischemic events (12).

The present study in patients with STEMI detected higher hs-CRP values $(\geq 0.5 \mathrm{mg} / \mathrm{dl})$ in older patients. This finding supports an earlier study which demonstrated that higher hs-CRP levels in the elderly were associated with a higher incidence of cardiovascular events compared with control subjects (13). In the present study there was a significant difference in mean hs-CRP values between patients with or without diabetes mellitus $(\mathrm{P}=0.029)$. A positive correlation of hs-CRP with blood sugar level was also observed $(r=0.165$, $\mathrm{P}=0.023$ ). This finding is in accordance with the study by Festa et al (14), which showed elevated serum hs-CRP levels in metabolic disorders, namely, hypertension, dyslipidemia, type 2 diabetes and insulin resistance, suggesting the 
Table IV. Association between hs-CRP and coronary angiogram data.

\begin{tabular}{|c|c|c|c|c|c|c|c|c|c|}
\hline \multirow[b]{2}{*}{ Parameter } & \multirow[b]{2}{*}{$\mathrm{N}$} & \multirow[b]{2}{*}{$\begin{array}{l}\text { Mean hs-CRP } \\
(\mathrm{mg} / \mathrm{dl})\end{array}$} & \multirow[b]{2}{*}{ SD } & \multirow[b]{2}{*}{$\begin{array}{l}\text { SE of } \\
\text { Mean }\end{array}$} & \multicolumn{2}{|c|}{$95 \% \mathrm{CI}$ for mean } & \multirow[b]{2}{*}{ Min } & \multirow[b]{2}{*}{$\operatorname{Max}$} & \multirow[b]{2}{*}{ P-value } \\
\hline & & & & & $\begin{array}{l}\text { Lower } \\
\text { bound }\end{array}$ & $\begin{array}{l}\text { Upper } \\
\text { bound }\end{array}$ & & & \\
\hline CAD & & & & & & & & & 0.625 \\
\hline SVD & 43 & 0.56 & 0.47 & 0.07 & 0.42 & 0.71 & 0.03 & 2.09 & \\
\hline DVD & 25 & 0.72 & 0.71 & 0.14 & 0.43 & 1.01 & 0.01 & 2.77 & \\
\hline TVD & 7 & 0.97 & 1.08 & 0.41 & -0.03 & 1.97 & 0.08 & 3.34 & \\
\hline Insignificant CAD & 12 & 0.76 & 1.28 & 0.37 & -0.05 & 1.57 & 0.04 & 4.67 & \\
\hline No. of vessels & & & & & & & & & 0.072 \\
\hline 1 & 33 & 0.54 & 0.82 & 0.14 & 0.25 & 0.83 & 0.03 & 4.67 & \\
\hline 2 & 32 & 0.68 & 0.56 & 0.10 & 0.47 & 0.88 & 0.01 & 2.09 & \\
\hline 3 & 22 & 0.85 & 0.84 & 0.18 & 0.48 & 1.22 & 0.08 & 3.34 & \\
\hline LMCA & & & & & & & & & 0.409 \\
\hline Unaffected & 84 & 0.65 & 0.72 & 0.08 & 0.49 & 0.81 & 0.01 & 4.67 & \\
\hline Affected & 3 & 1.20 & 1.38 & 0.79 & -2.22 & 4.61 & 0.22 & 2.77 & \\
\hline Thrombus & & & & & & & & & 0.979 \\
\hline Absent & 69 & 0.66 & 0.74 & 0.09 & 0.48 & 0.84 & 0.02 & 4.67 & \\
\hline Affected & 18 & 0.71 & 0.76 & 0.18 & 0.33 & 1.08 & 0.01 & 2.77 & \\
\hline Calcification & & & & & & & & & 0.358 \\
\hline Absent & 54 & 0.61 & 0.72 & 0.10 & 0.42 & 0.81 & 0.02 & 4.67 & \\
\hline Affected & 33 & 0.76 & 0.78 & 0.14 & 0.48 & 1.04 & 0.01 & 3.34 & \\
\hline Syntax score & & & & & & & & & 0.079 \\
\hline Mild CAD & 75 & 0.59 & 0.65 & 0.07 & 0.44 & 0.73 & 0.01 & 4.67 & \\
\hline Moderate CAD & 9 & 0.89 & 0.68 & 0.23 & 0.37 & 1.41 & 0.20 & 2.09 & \\
\hline Severe CAD & 3 & 2.11 & 1.66 & 0.96 & -2.02 & 6.24 & 0.22 & 3.34 & \\
\hline In-hospital mortality & & & & & & & & & 0.171 \\
\hline Absent & 174 & 0.70 & 0.81 & 0.06 & 0.58 & 0.83 & 0.01 & 4.98 & \\
\hline Affected & 16 & 0.74 & 0.47 & 0.12 & 0.49 & 0.99 & 0.21 & 1.80 & \\
\hline 30 day mortality & & & & & & & & & - \\
\hline Absent & 175 & 0.71 & 0.81 & 0.06 & 0.59 & 0.83 & 0.01 & 4.98 & \\
\hline Affected & 1 & 0.31 & - & - & - & - & 0.31 & 0.31 & \\
\hline
\end{tabular}

hs-CRP, high-sensitivity C-reactive protein; SD, standard deviation; SE, standard error; CI, confidence interval; CAD, coronary artery disease; SVD, single vessel disease; DVD, double vessel disease; TVD, triple vessel disease; LMCA, left main coronary artery.

involvement of a low-grade systemic inflammation in these disorders. In the present study, even though higher hs-CRP values were detected with the presence of hypertension, smoking, higher total cholesterol level, higher low-density lipoprotein (LDL) level, higher TIMI score and presence of LV dysfunction compared with the absence of these factors, no statistically significant difference in mean hs-CRP values was recorded. This could be due to low sample size and/or indicate the value of hs-CRP as an additive risk factor to the traditional risk factors already present as shown by earlier studies (14-17). This indicates that hs-CRP could help in the identification of patients at risk of cardiovascular events, even those previously classified as having low or intermediate risk.

Killip class in AMI is used to stratify patients with symptoms and/or signs of heart failure and predict in-hospital mortality (Killip class I has 6\%, whereas Killip class IV has $60 \%$ in-hospital mortality) (18). The present study demonstrated a statistically significant difference in mean hs-CRP concentrations among patients with different Killip classes (0.62 mg/dl in Killip class I vs. $0.98 \mathrm{mg} / \mathrm{ml}$ in Killip class IV; $\mathrm{P}<0.05)$. This finding also supports an earlier study by Suleiman et al (19), in which it was reported that patients with acute MI had CRP levels in the upper quartile, were older, had higher baseline creatinine levels and had a greater history of heart failure.

TIMI score in AMI is a convenient, simple bedside risk scoring system for predicting 30-day mortality at presentation for fibrinolytic-eligible patients with STEMI using the In TIME-II trial database (TIMI risk score 0 has mortality $0.8 \%$, where as TIMI score $>8$ has mortality $35.9 \%$ ) (20). In the 
Table V. Coronary angiogram findings with respect to hs-CRP value.

\begin{tabular}{|c|c|c|c|c|c|c|c|}
\hline \multirow[b]{2}{*}{ Parameter } & \multicolumn{2}{|c|}{$\begin{array}{c}\text { hs-CRP } \\
<0.5 \mathrm{mg} / \mathrm{dl}\end{array}$} & \multicolumn{2}{|c|}{$\begin{array}{c}\text { hs-CRP } \\
\geq 0.5 \mathrm{mg} / \mathrm{dl}\end{array}$} & \multirow[b]{2}{*}{ Total } & \multirow[b]{2}{*}{$\chi^{2}$} & \multirow[b]{2}{*}{ P-value } \\
\hline & $\mathrm{N}$ & $\%$ & $\mathrm{~N}$ & $\%$ & & & \\
\hline CAD & & & & & & 4.143 & 0.216 \\
\hline SVD & 23 & 53 & 20 & 47 & 43 & & \\
\hline DVD & 12 & 48 & 13 & 52 & 25 & & \\
\hline TVD & 1 & 14 & 6 & 86 & 7 & & \\
\hline Insignificant CAD & 7 & 58 & 5 & 42 & 12 & & \\
\hline No. of vessels & & & & & & 7.065 & 0.029 \\
\hline 1 & 22 & 67 & 11 & 33 & 33 & & \\
\hline 2 & 14 & 44 & 18 & 56 & 32 & & \\
\hline 3 & 7 & 32 & 15 & 68 & 22 & & \\
\hline LMCA & & & & & & 0.322 & 0.570 \\
\hline Unaffected & 42 & 50 & 42 & 50 & 84 & & \\
\hline Affected & 1 & 33 & 2 & 67 & 3 & & \\
\hline Thrombus & & & & & & 0.341 & 0.559 \\
\hline Absent & 33 & 48 & 36 & 52 & 69 & & \\
\hline Affected & 10 & 56 & 8 & 44 & 18 & & \\
\hline Calcification & & & & & & 1.043 & 0.307 \\
\hline Absent & 29 & 54 & 25 & 46 & 54 & & \\
\hline Affected & 14 & 42 & 19 & 58 & 33 & & \\
\hline Syntax score & & & & & & 3.433 & 0.180 \\
\hline Mild CAD & 40 & 53 & 35 & 47 & 75 & & \\
\hline Moderate CAD & 2 & 22 & 7 & 78 & 9 & & \\
\hline Severe CAD & 1 & 33 & 2 & 67 & 3 & & \\
\hline
\end{tabular}

hs-CRP, high-sensitivity C-reactive protein; CAD, coronary artery disease; SVD, single vessel disease; DVD, double vessel disease; TVD, triple vessel disease; LMCA, left main coronary artery.

Table VI. Syntax score and number of affected vessels with respect to hs-CRP value.

\begin{tabular}{|c|c|c|c|c|c|c|}
\hline \multirow[b]{2}{*}{ Parameter } & \multicolumn{2}{|c|}{ hs-CRP $<0.5 \mathrm{mg} / \mathrm{dl}$} & \multicolumn{2}{|c|}{ hs-CRP $\geq 0.5 \mathrm{mg} / \mathrm{dl}$} & \multirow[b]{2}{*}{ Mean difference } & \multirow[b]{2}{*}{ P-value } \\
\hline & Mean & $\mathrm{SD}$ & Mean & $\mathrm{SD}$ & & \\
\hline No. of vessels & 1.65 & 0.75 & 2.09 & 0.77 & -0.440 & 0.009 \\
\hline Syntax score & 11.06 & 7.54 & 15.26 & 8.84 & -4.203 & 0.019 \\
\hline
\end{tabular}

hs-CRP, high-sensitivity C-reactive protein; SD, standard deviation.

present study a weak correlation of hs-CRP with TIMI score ( $\mathrm{r}=0.131, \mathrm{P}=0.073)$ was observed. Foussas et al (21) suggested the prognostic usefulness of hs-CRP levels along with the well-validated TIMI risk score for STEMI and non-STEMI.

Increased hs-CRP values correlate with other risk scores in ACS. In one study, Schiele et al (22) demonstrated an independent predictive role of CRP in ACS patients; patients in the highest quartile of CRP showed increased mortality rates at 30 days of follow-up. Hence, in a setting of ACS, the
hs-CRP value may be combined with well-validated risks such as TIMI and Global Registry of Acute Coronary Events scores, and helps in better predicting adverse events. In the present study 77 (40\%) patients with acute STEMI admitted with a window period of $0-6 \mathrm{~h}$ had hs-CRP values $<0.5 \mathrm{mg}$ / $\mathrm{dl}$, and there was no significant difference in hs-CRP values between different types of MI. These findings accorded well with the study by Cristell et al (23), which reported that $41 \%$ of patients admitted $<6 \mathrm{~h}$ from symptom onset had hs-CRP 
Table VII. In-hospital complications.

\begin{tabular}{|c|c|c|c|c|c|c|c|c|c|}
\hline \multirow[b]{2}{*}{ Complications } & \multirow[b]{2}{*}{$\mathrm{N}$} & \multirow[b]{2}{*}{$\begin{array}{l}\text { Mean hs-CRP } \\
(\mathrm{mg} / \mathrm{dl})\end{array}$} & \multirow[b]{2}{*}{$\mathrm{SD}$} & \multirow[b]{2}{*}{$\begin{array}{l}\text { SE of } \\
\text { mean }\end{array}$} & \multicolumn{2}{|c|}{$95 \% \mathrm{CI}$ for mean } & \multirow[b]{2}{*}{ Min } & \multirow[b]{2}{*}{ Max } & \multirow[b]{2}{*}{ P-value } \\
\hline & & & & & $\begin{array}{l}\text { Lower } \\
\text { bound }\end{array}$ & $\begin{array}{l}\text { Upper } \\
\text { bound }\end{array}$ & & & \\
\hline LV dysfunction & & & & & & & & & 0.328 \\
\hline Absent & 25 & 0.67 & 1.00 & 0.20 & 0.25 & 1.08 & 0.02 & 4.98 & \\
\hline Affected & 165 & 0.71 & 0.76 & 0.06 & 0.60 & 0.83 & 0.01 & 4.67 & \\
\hline Heart failure & & & & & & & & & 0.267 \\
\hline Absent & 160 & 0.70 & 0.82 & 0.06 & 0.57 & 0.83 & 0.01 & 4.98 & \\
\hline Affected & 30 & 0.74 & 0.61 & 0.11 & 0.51 & 0.97 & 0.05 & 2.53 & \\
\hline Shock & & & & & & & & & 0.094 \\
\hline Absent & 178 & 0.70 & 0.81 & 0.06 & 0.58 & 0.82 & 0.01 & 4.98 & \\
\hline Affected & 12 & 0.85 & 0.48 & 0.14 & 0.54 & 1.15 & 0.14 & 1.80 & \\
\hline Acute MR & & & & & & & & & 0.031 \\
\hline Absent & 176 & 0.69 & 0.80 & 0.06 & 0.57 & 0.81 & 0.01 & 4.98 & \\
\hline Affected & 14 & 0.97 & 0.67 & 0.18 & 0.58 & 1.35 & 0.20 & 2.53 & \\
\hline VSR & & & & & & & & & - \\
\hline Absent & 188 & 0.71 & 0.79 & 0.06 & 0.60 & 0.82 & 0.01 & 4.98 & \\
\hline Affected & 1 & 0.33 & - & - & - & - & 0.33 & 0.33 & \\
\hline Bradyarrythmias & & & & & & & & & 0.349 \\
\hline Absent & 176 & 0.70 & 0.81 & 0.06 & 0.58 & 0.82 & 0.01 & 4.98 & \\
\hline Affected & 14 & 0.76 & 0.55 & 0.15 & 0.44 & 1.07 & 0.14 & 1.80 & \\
\hline Tachyarrythmias & & & & & & & & & 0.120 \\
\hline Absent & 179 & 0.70 & 0.80 & 0.06 & 0.58 & 0.81 & 0.01 & 4.98 & \\
\hline Affected & 11 & 0.90 & 0.64 & 0.19 & 0.47 & 1.33 & 0.20 & 2.26 & \\
\hline Recurrent angina & & & & & & & & & 0.459 \\
\hline Absent & 187 & 0.71 & 0.80 & 0.06 & 0.59 & 0.82 & 0.01 & 4.98 & \\
\hline Affected & 3 & 0.74 & 0.32 & 0.18 & -0.06 & 1.53 & 0.39 & 1.02 & \\
\hline LV thrombus & & & & & & & & & 0.097 \\
\hline Absent & 188 & 0.71 & 0.79 & 0.06 & 0.60 & 0.83 & 0.01 & 4.98 & \\
\hline Affected & 2 & 0.13 & 0.11 & 0.08 & -0.83 & 1.08 & 0.05 & 0.20 & \\
\hline
\end{tabular}

hs-CRP, high-sensitivity C-reactive protein; SD, standard deviation; SE, standard error; CI, confidence interval; LV, left ventricular; MR, mitral regurgitation; VSR, ventrical septal rupture.

values $<2 \mathrm{mg} / \mathrm{l}(23)$. The same study showed a wide dispersion of hs-CRP values, largely overlapping with those of control subjects and authors could not explain the exact reason for hs-CRP values $<2 \mathrm{mg} / 1$ in $41 \%$ of STEMI patients. Similar findings of a very wide dispersion of hs-CRP values were reported in the control subjects of the PROVE IT-TIMI 22 study, which was found to be unrelated to LDL cholesterol levels (24). In the present study, with regard to window period, a greater number of patients presented with hs-CRP values $>0.5 \mathrm{mg}$ beyond $6 \mathrm{~h}(\mathrm{P}=0.05)$. The mechanism for CRP elevation and its predictive value for different outcomes in patients with ACS depend critically upon the clinical setting and time of measurement $(8,25)$.

The Syntax score is a comprehensive angiographic scoring system derived entirely from the coronary anatomy and lesion characteristics (26-28). In the present study, the hs-CRP values were shown to be positively correlated with
Syntax score $(r=0.285, P=0.007)$. Higher Syntax scores were associated with high hs-CRP values (mean hs-CRP value for a score $\leq 22$ was 0.59 vs. $2.11 \mathrm{mg} / \mathrm{dl}$ for a score $\geq 33$ ). To the best of our knowledge, there are no previous studies correlating hs-CRP with Syntax score in STEMI. However, a few studies have correlated it with the presence and extent of severity of CAD in ACS; the present study findings corroborate earlier studies, which have shown a correlation between CRP and the severity of CAD $(29,30)$.

Zebrack et al (29) reported a weak association between CRP and CAD score. In view of their result, they suggested that CRP could determine primary plaque properties (that is, inflammation and instability), whereas CAD score could identify the extent of the atherosclerotic plaque.

In an another study, Arroyo-Espliguerol et al (31) showed a significantly higher hs-CRP value in patients with ACS compared with chronic stable angina $(\mathrm{P}=0.004)$ and a 
Table VIII. hs-CRP and mortality.

\begin{tabular}{|c|c|c|c|c|c|c|c|}
\hline \multirow[b]{2}{*}{ Mortality } & \multicolumn{2}{|c|}{$\begin{array}{c}\text { hs-CRP } \\
<0.5 \mathrm{mg} / \mathrm{dl}\end{array}$} & \multicolumn{2}{|c|}{$\begin{array}{c}\text { hs-CRP } \\
\geq 0.5 \mathrm{mg} / \mathrm{dl}\end{array}$} & \multirow[b]{2}{*}{ Total } & \multirow[b]{2}{*}{$\chi^{2}$} & \multirow[b]{2}{*}{ P-value } \\
\hline & $\mathrm{N}$ & $\%$ & $\mathrm{~N}$ & $\%$ & & & \\
\hline In-hospital mortality & & & & & & 0.273 & 0.601 \\
\hline Absent & 88 & 51 & 86 & 49 & 174 & & \\
\hline Affected & 7 & 44 & 9 & 56 & 16 & & \\
\hline 30 day mortality & & & & & & 1.006 & 0.316 \\
\hline Absent & 87 & 50 & 88 & 50 & 175 & & \\
\hline Affected & 1 & 100 & 0 & 0 & 1 & & \\
\hline
\end{tabular}

hs-CRP, high-sensitivity C-reactive protein.

correlation with complex angiographic lesions $(\mathrm{P}=0.001)$. Similar findings were reported by Azar et al (32) in an angiographic study. However, Rifai et al (33) did not find any significant association between inflammatory markers and CAD.

With regard to various complications, although mean hs-CRP values were higher in the presence of various complications, there was no significant difference of hs-CRP values according to the presence or absence of a complication, with the exception of patients who developed mitral regurgitation, where there was a significant difference between mean hs-CRP values. It is possible that the values for various complications may reach statistical significance when a larger sample size is used. In the present study there was no statistically significant difference observed between mean hs-CRP values in patients with or without in-hospital mortality; this could be explained by the small study sample size. However, there are multiple studies which have demonstrated an increased mortality rate in patients with STEMI and increased hs-CRP (34-38).

The cross-sectional nature and relatively small study sample are obvious limitations of the present study. Because of this, the study could not establish causality and could only establish an association. With difficulties in confirming the external validity of the results, the conclusions derived from this study must be considered preliminary and hypothesis-generating.

Inflammation plays an important role in atherothrombosis, and the measurement of inflammatory markers, specifically hs-CRP, might provide a novel method for detecting individuals with a high risk of plaque rupture in ACS. hs-CRP measurement is a valuable tool in STEMI patients. This study highlighted the association of increased hs-CRP value with higher Killip class, higher TIMI score, and most importantly with higher Syntax score, which indicates an overall atherosclerotic burden of coronary arteries. The present study has also demonstrated an association of higher hs-CRP values with increased hospital complications, and increased short- and long-term mortality. It is also considered that the highlighted role of inflammation in this study will increase understanding of atherosclerosis in general and should have clinical applications in therapy targeting for this serious disease.

\section{Acknowledgements}

The authors extend their appreciation to the Deanship of Scientific Research at King Saud University for funding this work through research group project number RGP-215.

\section{References}

1. Armani A and Becker RC: The biology, utilization and attenuation of C-reactive protein in cardiovascular disease: Part I. Am Heart J 149: 971-976, 2005.

2. Braunwald E: Shattuck lecture - cardiovascular medicine at the turn of the millennium: Triumphs, concerns and opportunities. N Engl J Med 337: 1360-1369, 1997.

3. Libbey P, Ridker PM and Maseri A: Inflammation and atherosclerosis. Circulation 105: 1135-1143, 2002.

4. Yeh ET and Willerson JT: Coming of age of C-reactive protein: Using inflammation markers in cardiology. Circulation 107: 370-371, 2003.

5. Rifai $\mathrm{N}$ and Ridker PM: High sensitive C-reactive protein: A novel and promising marker of coronary heart disease. Clin Chem 47: 403-411, 2001.

6. Roberts WL, Moulton L, Law TC, Farrow G, Cooper-Anderson M, Savory $\mathrm{J}$ and Rifai N: Evaluation of nine automated high sensitive C-reactive protein methods: Implication for clinical and epidemiological applications. Part 2. Clin Chem 47: 418-425, 2001.

7. Ridker PM, Glynn RJ and Hennekens CH: C-reactive protein adds to the predictive value of total and HDL cholesterol in determining risk of first myocardial infarction. Circulation 97: 2007-2011, 1998.

8. Ridker PM, Hennekens CH, Buring JE and Rifai N: C-reactive protein and other markers of inflammation in the prediction of cardiovascular disease in women. N Engl J Med 342: 836-843, 2000.

9. Thygesen K, Alpert JS, Jaffe AS, Simoons ML, Chaitman BR, White HD; Joint ESC/ACCF/AHA/WHF Task Force for the Universal Definition of Myocardial Infarction, Katus HA, Lindahl B, Morrow DA, et al: Third universal definition of myocardial infarction. Circulation 126: 2020-2035, 2012.

10. Antman Elliott M, Marc C, Peter JLMB, Carolyn HM, Thomas H, Gary P, Branco M, Ramon C, David R and Eugene B: The TIMI risk score for unstable angina/non-ST elevation MI: a method for prognostication and therapeutic decision making. JAMA 284: 835-842, 2000.

11. Serruys PW, Morice MC, Kappetein AP, Colombo A, Holmes DR, Mack MJ, Ståhle E, Feldman TE, van den Brand M, Bass EJ, et al: Percutaneous coronary intervention versus coronary-artery bypass grafting for severe coronary artery disease. N Engl J Med 360: 961-972, 2009.

12. Biasucci LM, Liuzzo G, Grillo RL, Caligiuri G, Rebuzzi AG, Buffon A, Summaria F, Ginnetti F, Fadda G and Maseri A: Elevated levels of C-reactive protein at discharge in patients with unstable angina predict recurrent instability. Circulation 99: 855-860, 1999. 
13. Tracy RP, Lemaitre RN, Psaty BM, Ives DG, Evans RW, Cushman M, Meilahn EN and Kuller LH: Relationship of C-reactive protein to risk of cardiovascular disease in the elderly: Results from the Cardiovascular Health Study and the Rura Health Promotion Project. Arterioscler Thromb Vasc Biol 17: 1121-1127, 1997.

14. Festa A, D'Agostino R Jr, Howard G, Mykkänen L, Tracy RP and Haffner SM: Chronic subclinical inflammation as part of the insulin resistance syndrome: The insulin resistance atherosclerosis study (IRAS). Circulation 102: 42-47, 2000.

15. Fröhlich M, Imhof A, Berg G, Hutchinson WL, Pepys MB, Boeing $\mathrm{H}$, Muche R, Brenner $\mathrm{H}$ and Koenig W: Association between $\mathrm{C}$-reactive protein and features of the metabolic syndrome: A population-based study. Diabetes Care 23: 1835-1839, 2000

16. Pickup JC, Mattock MB, Chusney GD and Burt D: NIDDM as a disease of the innate immune system: Association of acute-phase reactants and interleukin-6 with metabolic syndrome $X$. Diabetologia 40: 1286-1292, 1997.

17. Yudkin JS, Kumari M, Humphries SE and Mohamed-Ali V: Inflammation, obesity, stress and coronary heart disease: Is interleukin-6 the link? Atherosclerosis 148: 209-214, 2000.

18. Hass E, Yang EH, Gersh BJ and O'Rourke RA: ST-segment elevation myocardial infarction. In: Hurst's The Heart. Fuster V, Walsh RA. and Harrington RA (eds). 13th edition. McGraw Hill, New York, pp1356-1357, 2011.

19. Suleiman M, Khatib R, Agmon Y, Mahamid R, Boulos M, Kapeliovich M, Levy Y, Beyar R, Markiewicz W, Hammerman H and Aronson D: Early inflammation and risk of long-term development of heart failure and mortality in survivors of acute myocardial infarction: J Am Coll Cardiol 47: 962-968, 2006.

20. Morrow DA, Antman EM, Charlesworth A, Cairns R, Murphy SA, de Lemos JA, Giugliano RP, McCabe CH and Braunwald E: TIMI risk score for ST elevation myocardial infarction: A convenient, bedside, clinical score for risk assessment at presentation: An intravenous nPA for treatment of infarcting myocardium early II trial substudy. Circulation 102: 2031-2037, 2000.

21. Foussas SG, Zairis MN, Lyras AG, Patsourakos NG, Tsirimpis VG, Katsaros K, Beldekos DJ, Handanis SM, Mytas DZ, Karidis KS, et al: Early prognostic usefulness of C-reactive protein added to the Thrombolysis In Myocardial Infarction risk score in acute coronary syndromes. Am J Cardiol 96: 533-537, 2005.

22. Schiele F, Meneveau N, Seronde MF, Chopard R, Descotes-Genon V, Dutheil J and Bassand JP; Reseau de Cardiologie de Franche Comte: C-reactive protein improves risk prediction in patients with acute coronary syndromes. Eur Heart J 31: 290-297, 2010.

23. Cristell N, Cianflone D, Durante A, Ammirati E, Vanuzzo D, Banfi M, Calori G, Latib A, Crea F, Marenzi G, et al: High-sensitivity C-reactive protein is within normal levels at the very onset of first ST-segment elevation acute myocardial infarction in $41 \%$ of cases: a multiethnic case-control study. J Am Coll Cardiol 58: 2654-2661, 2011.

24. Casas JP, Shah T, Hingorani AD, Danesh J and Pepys MB: C-reactive protein and coronary heart disease: A critical review. J Intern Med 264: 295-314, 2008.

25. Ridker PM, Cushman M, Stampfer MJ, Tracy RP and Hennekens CH: Inflammation, aspirin and the risk of cardiovascular disease in apparently healthy men. N Engl J Med 336 973-979, 1997.
26. Sianos G, Morel MA, Kappetein AP, Morice MC, Colombo A, Dawkins K, van den Brand M, Van Dyck N, Russell ME, Mohr FW and Serruys PW: The SYNTAX score: An angiographic tool grading the complexity of coronary artery disease. EuroIntervention 1: 219-227, 2005.

27. Serruys PW, Onuma Y, Garg S, Sarno G, van den Brand M, Kappetein AP, Van Dyck N, Mack M, Holmes D, Feldman T, et al: Assessment of the SYNTAX score in the Syntax study. EuroIntervention 5: 50-56, 2009.

28. SYNTAX working group. SYNTAX score calculator. http:// www.syntaxscore.com. Accessed November 2009.

29. Zebrack JS, Muhlestein JB, Horne BD and Anderson JL; Intermountain Heart Collaboration Study Group: C-reactive protein and angiographic coronary artery disease: Independent and additive predictors of risk in subjects with angina. J Am Coll Cardiol 39: 632-637, 2002.

30. Tataru MC, Heinrich J, Junker R, Schulte H, von Eckardstein A, Assmann G and Koehler E: C-reactive protein and the severity of atherosclerosis in myocardial infarction patients with stable angina pectoris. Eur Heart J 21: 1000-1008, 2000.

31. Arroyo-Espliguero R, Avanzas P, Cosín-Sales J, Aldama G, Pizzi C and Kaski JC: C-reactive protein elevation and disease activity in patients with coronary artery disease. Eur Heart J 25: 401-408, 2004

32. Azar RR, Aoun G, Fram DB, Waters DD, Wu AH and Kiernan FJ: Relation of C-reactive protein to extent and severity of coronary narrowing in patients with stable angina pectoris or abnormal exercise tests. Am J Cardiol 86: 205-207, 2000.

33. Rifai N, Joubran R, Yu H, Asmi M and Jouma M: Inflammatory markers in men with angiographically documented coronary heart disease. Clin Chem 45: 1967-1973, 1999.

34. Tanaka A, Shimada K, Sano T, Namba M, Sakamoto T, Nishida Y, Kawarabayashi T, Fukuda D and Yoshikawa J: Multiple plaque rupture and C-reactive protein in acute myocardial infarction. J Am Coll Cardiol 45: 1594-1599, 2005.

35. Morrow DA, Rifai N, Antman EM, Weiner DL, McCabe CH, Cannon CP and Braunwald E: C-reactive protein is a potent predictor of mortality independently of and in combination with troponin $\mathrm{T}$ in acute coronary syndromes: A TIMI 11A substudy. Thrombolysis in Myocardial Infarction. J Am Coll Cardiol 31: 1460-1465, 1998

36. Lindahl B, Toss H, Siegbahn A, Venge P and Wallentin L; FRISC Study Group: Markers of myocardial damage and inflammation in relation to long-term mortality in unstable coronary artery disease. Fragmin during instability in coronary artery disease. $\mathrm{N}$ Engl J Med 343: 1139-1147, 2000.

37. Mueller C, Buettner HJ, Hodgson JM, Marsch S, Perruchoud AP, Roskamm H and Neumann FJ: Inflammation and long-term mortality after non-ST-elevation acute coronary syndrome treated with a very early invasive strategy in 1042 consecutive patients. Circulation 105: 1412-1415, 2002.

38. James SK, Armstong P, Barnathan E, Califf R, Lindahl B, Siegbahn A, Simoons ML, Topol EJ, Venge P and Wallentin L; GUSTO-IV-ACS Investigators: Troponin and C-reactive protein have different relations to subsequent mortality and myocardial infarction after acute coronary syndrome: A GUSTO-IV substudy. J Am Coll Cardiol 41: 916-924, 2003. 